utilise twenty races for obtaining the common racial mean. His method of discussion is based on the safe ground that, if racial variability be associated with individual variability, whenever any specified character is more variable than another specified character in the one, it will (on the whole) be more variable in the other also. Therefore if the reduced quartiles of the two characters, in the series of means of races, be called $A$ and $B$. and those in that of the individuals of any given race be called $a$ and $b$, then if $\mathrm{A}$ be greater than $a, \mathrm{~B}$ would (on the whole) be greater than $\dot{b}$, and conversely. More briefly and fully, if the signs of the differences (A-B) and $(a-b)$ are alike, the evidence, so far as it goes, favours the suggested idea of a connection between racial and individual variability; if the signs are different it discountenances it.

The test is rough, but is of value when applied on a considerable scale with concurrent results, as in the present instance. The eighteen characters admit of $\frac{1}{2}(18 \times 17)=153$ different pairs of combinations of the form $(a-b)$, in each of the eight races; that is I224 pairs in all. Each of these has been compared with its associated pair in the series of means of races, of which there are altogether $\frac{1}{2}(8 \times 7)=28$. The result is that in seven out of the eight races the cases testifying to the existence of the suggested association are from twice to thrice as numerous as the others, and in the eighth race they are $\mathrm{I} \frac{1}{2}$ times as numerous. Nay more, there is some evidence that the most variable characters in the one, are the most variable in the other. This conclusion is corroborated by three other inquiries of the same kind, two into rodents and one into carnivoræ. The erro introduced by the strained assumption, that the ordinary law of frequency holds good for the series of means of allied races, does not seem likely to invalidate the general conclusion to a serious extent. It therefore appears that Mr. Brewster has provisionally established his thesis that whenever any specified character varies much in individuals of the same race, it is probable that it will be found to vary much in "allied" races, and conversely.

\section{ON THE CONSTITUTION OF THE ELECTRIC} SPARK. ${ }^{1}$

IF a Leyden jar is discharged through metal electrodes, and the spectrum of the spark is examined, it is found that the metallic lines are not confined to the immediate neighbourhood of the poles, but are seen sometimes in the centre of the spark, several millimetres away from the electrodes, from which they must have been projected with considerable velocity.

It has always seemed to me to be a problem of interest, to measure the velocity of projection. A knowledge of it may teach us something concerning the mechanism of electric sparks and, in addition, we may hope to obtain information on some important points in spectrum analysis, which are at present under discussion. Thus, for instance, if the speed with which a molecule is pushed forward into the centre of the spark depends on molecular weight, we may separate from each other those lines of the spectrum which belong to different molecular combinations. For many years past I had made various unsuccessful attempts to deal with this problem, when I became acquainted with the elegant method, used by Prof. Dixon in some of his recent experiments, in which a photograph is taken on a film fixed to the rim of a rapidly revolving wheel, of which the speed may easily be made sufficiently large to measure velocities of moving luminous particles up to 2000 metres. This number might be doubled or trebled with improved appliances.

The experiments were conducted by Mr. Gustav Hemsalech, to whose care and skill their success is largely due. Without entering into a detailed description of the apparatus, it will be sufficient to say that the photographs, which I now submit to the Section, were taken on a film moving with a linear speed of about $80 \frac{\text { metres }}{\text { second }}$ in a direction at right angles to the slit of the spectroscope. The lines of the metal appear inclined instead of straight, in consequence of the finite velocity of the luminous molecules. The air lines, on the other hand, though slightly broadened, remain straight. The sparks were taken from five large Leyden jars, charged by means of a Voss machine. Each

1 By Prof. Arthur Schuster, F.R.S. (Read before Section A of the British Associaticn at the 'J'oronto meeting )

NO. I 462 , VOL. 57$]$ single spark produces a good spectrum, reaching approximately from $\lambda=5000$ to $\lambda=4000$

One of the photographs, in which zinc poles were used, shows that the velocity of the molecules is gradually diminishing as they move away from the pole.' Close to it the speed seems very great, the average velocity up to a distance of about one millimetre being about 2000 metres per second. At a distance of four millimetres the speed is reduced to something like 400 .

In another experiment one pole was zinc, while the other was bismuth. Some bismuth lines are found to be decidedly more curved than those of zinc, indicating a smaller velocity. But the line of bismuth, which lies at 4560 , seems almost straight.

When the poles are moistened with a solution of calcium chloride interesting results are obtained, the calcium line at 4226 being more inclined than $\mathrm{H}$ and $\mathrm{K}$.

The experiments were made with comparatively rough appliances, but a more perfect apparatus is in course of construction; and the author hopes to continue the research in conjunction with Mr. Hemsalech.

\section{PHYSTOLOGY AT THE BRITISH ASSOCIATION.}

THE section of Physiology at the British Association meeting at Toronto was a large and active one. Under the presidency of Prof. Michael Foster a large body of physiologists attended in about equal numbers from the east and west of the Atlantic. Meetings were held on the Thursday and Friday, August 19 and 20 , and on the Monday, Tuesday and Wednesday, August 23, 24 and 25 .

Among those present were Messrs. Bowditch, Boyce, Cushny, Crookshank, Gaskell, IFalliburton, Huber, Huerthle, Lee, Loeb, Lombard, Billings, Lister, Macallum, Osler, Wesley Mills, Noël Paton, Porter, Waymouth Reid, Sherrington, G. N. Stewart, Anderson Stuart, Kellog, W. H. Thompson, Charles Richet, Waller, Welby, Shore, MacAlister, O. Grunbaum, Baldwin, Braun, Reynolds Green, Meldola, and A. S. Grunbaum.

Prominent among proceedings in the Section were the follow-

Prof. Bowditch read a paper on the physiology of unstriped muscular tissue as exemplified in the wall of the stomach of the frog. The rhythmic contractility of the tissue is well seen. When the organ or a strip of it is fitted to a recording apparatus, in the majority of instances after a lapse of not more than three hours, contractions of rhythmic recurrence are registered. Often two or more sets of rhythmic contractions are superposed. This may be best accounted for by supposing the musclecells to contract not all together but in tivo or more groups. In the discussion Prof. Sherrington referred to experiments published by him in which had been recorded contractions of the urinary bladder removed from freshly-killed monkeys and placed in warm normal saline solution, while connected with a volume recorder. In these the isolated bladder began to "beat" almust at once, and continued "beating" for an hour or less, at rate somewhat quicker than once a minute. The contractions thus obtained from the viscus only exceptionally displayed the compound character shown in Prof. Bowditch's curves.

Prof. Carl Huber brought forward observations on the cells of the sympathetic system of vertebrates. In Amphibia nearly all sympathetic cells are unipolar. In other vertebrates the prevailing type is multipolar. All sympathetic neurons have one axon only. The dendrites form a network between the cellbodies of the neurons constituting a ganglion. The axon of each sympathetic nerve-cell becomes either a non-myelinated nerve-fibre (grey fibre) or a fine myelinated nerve-fibre. The fibres of the white rami are axons of cells lying within the spinal cord, and these axons reach the sympathetic ganglia through the white rami, and in the ganglia undergo branching, ending in baskets which enclose the perikarya of the sympathetic neurons. In mammalia and birds the circumcellular baskets are comparatively simple networks of varicose fibrillæ. In Reptilia and Amphibia instead of simple end baskets the fibre is spirally wound and completely contorted. This is the explanation of Beale's spiral fibre in the sympathetic cells of the frog figured by him forty years ago. In all vertebrates the pericellular baskets are intracapsular. Langley and his pupils have shown that an impulse travelling along a spino-sympathetic efferent chain may be blocked in a sympathetic ganglion by the injection of nicotin. This has been applied to practically all regions of the 
sympathetic by studies carried out on Langley and Sherrington's pilomotor nerve-system. Huber suggests that the nicotin paralyses not the perikarya of the sympathetic neurons, but rather the end baskets of the pre-ganglionic fibres.

Prof. G. N. Stewart brought forward the results of the application of a new modification of his electrical method of determining speed of blood flow to the question of the output of the mammalian heart. The method depends upon the change of resistance to the passage of electric currents through the blood in an artery or vein brought about by injection of blood serum. Serum conducts better than does entire blood. When the blood admixed with serum reaches the point of blood vessel under examination, electric currents previously balanced by a Wheatstone bridge are thrown out of balance, and it is arranged that a telephone shall announce this event. A specimen of blood is drawn from the corresponding artery in the other limb, and from this is determined the amount of serum which must be added to the normal blood to make its resistance equal to that collected during the passage of the mixture. The output of the heart can, from these data, be determined for the period of injection, and consequently by knowing the pulse-frequency be determined for a single beat. In the dog it is about $2 \cdot 3$ c.c. per second per kilogramme of body-weight.

Prof. Townsend Porter gave an account of observations made upon a strip of the muscle of the apex of the dog's ventricle. The strip is left attached only by a band of pericardium, and yet exhibits in this isolated state rhythmic spontaneous beats. The rhythm of its beat is slow but perfectly regular; it is slower than the rhythm of the rest of the ventricle ; it, of course, persisted when the rest of the ventricle was arrested by excitation of the vagus nerve. The blood supply was maintained in the s'rip by means of an artery and vein in the pericardium. The isolation of the strip from the rest of the myocardium is found, even under microscopical examination, to be absolutely complete. The contractions of the isolated piece could not have been caused by excitation by the actioncurrent accompanying the systole of the rest of the ventricle, as the piece was raised freely in the air. The asynchronism of the beat of the isolated strip and rest of the ventricle prove the same thing, also that the strip was not discharged by nerves accompanying the blood vessels to the strip. Other experiments showed that if an artificial circulation of diluted blood be kept up through the extreme apex of the dog's heart excised and hung up on cannulae the isolated apex will be vigorously, coordinately, and regularly for several hours. Hence it is concluded that the apex and other parts of the mammalian hear possess spontaneous rhythmic contractility, that the cause of rhythmic contraction is not a single localised coordination centre, the mechanism of coordination, whatever it be, being present in all parts of the ventricle.

Prof. Porter also described experiments confirming MacWilliam's statement that the ventricle of the dog's heart can be recovered from fibrillary contractions. The entire heart of the cat can be readily made to recover even after long-continued and violent fibrillary contraction by free and steady periusion of fresh blood through the coronary vessels.

When a vein on the surface of the ventricle in situ in the living animal is incised and the heart slowed by vagus excitation, the flow from the cut vein is much increased during ventricular contraction. The contraction of the cardiac muscle compresses the veins, and to a less extent the arteries, in the substance of the heart. The systole aids the circulation of blood through the heart muscle. On the other hand, observations with minimum manometers on the veins of the heart give no support to the view that the heart acts on the coronary circulation to any extent as a suction-pump, although efficiently as a forcepump. A circulation through the vessels of Thebesius was demonstrated to be sufficient to keep up the rhythmic beat of the right venticle after the coronary arteries have been closed. Ringer's solution will not maintain the activity of the mammalian heart, but diluted fresh defibrinated blood will.

Prof. Waymouth Reid gave an account of his most recent researches on absorption in the intestine. Heidenhain demon strated the fact that the water, organic and inorganic solids, of serum introduced into the intestine, are absorbed. The experiment was devised in support of the theory that intestinal absorp tion is possible under conditions in which osmotic transfer is ex cluded, and it was found that even inspissated serum is absorbed. Heidenhain omitted to mcasure the hydrostatic pressure on either side of the intestinal membrane, so that the possibility of the result being due to filtration was not excluded ; and, indeed, the ancient filtration theory of Lieberkiihn has, with the necessary modern histological modifications, been revived of late by Hamburger. In the experiments now described, the animal's own serum (obtained by the centrifugal machine) was introduced into a loop of its intestine, and the hydrostatic pressure in the cavity of the experimental loop, and in a mesenteric vein pro. ceeding from a control loop, filled with "normal saline" solution, observed continuously during the course of the experiment. Prof. Reid now found that water, organic and inorganic solids, are absorbed against considerable excess of hydrostatic pressure in the blood-vessels. (Since the velocity of the blood-stream in capillaries is low, it is taken for granted that the pressure in the capillaries of the intestinal villi is not lower than that in a mesenteric vein at the border of the gut.) The results of his experiments are practically the same when all the lacteals leaving the experimental loop of intestine have been occluded by ligature.

Prof. Reid called attention to our present inability to explain the phenomena. Great difficulties are offered in the face of the following points, examined and proven by his experiments.

(I) Osmosis, filtration into the blood capillaries, or into the lacteals by the action of Briicke's "villus pump" are, it is considered, excluded by the conditions of the experiment.

(2) That the disappearance of the serum from the cavity of the gut is simply a matter of imbibition is in the highest degree improbable, because the cells must be, at the commencement of the experiment, soaked to the highest degree possible in those constituents of the animal's serum which they are capable of taking up.

(3) Electro-osmotic action is again improbable, because secreting membranes produce ingoing electrical currents as well as absorbing membranes; and, to apply such an hypothesis, it would be necessary to assume that the ingoing current of the cells is active in one case (absorption), the outgoing return current in the other (secretion) involving the further hypothesis of some valvular nature of protoplasm with higher "porosity" in the "in-out" direction in the absorbing, and the "out-in" direction in the secreting, membrane.

(4) Finally, any aspirating action of the blood current in the capillaries of the villi is negligible on account of the low velocity of the current in capillary districts of the circulation.

Prof. W. H. Thompson reported on experiments continued under the committee appointed to examine the effects of peptone and its precursors when introduced into the circulation. The experiments had dealt with the influence of pure peptone, of anti-peptone, and of hetero-albumose on coagulation, on bloodpressure, and on vasomotor irritability. Pure peptone was found to delay but not destroy the coagulability of blood, and to cause a fall in blood-pressure and to lower the vaso-muscular irritability. Antipeptone was found to hasten the coagulation of blood : it causes a very transient fall of arterial blood-pres. sure, which is immediately followed by a long-continued slight increase of blood-pressure above that present at commencement of the experiment. As to deutero-albumose this substance was not found to give constant results : in some experiments a marked retardation, in others a marked increase of rate of coagulation of blood ensued. It causes a considerable fall in the arterial blood-pressure. Further experiments are in progress on the subject.

Prof. Carl Huber brought forward an important and lengthy paper on the modes of ending of nerve-fibres in smooth, cardiac, and striated muscles. Regarding the last-mentioned it was urged that the nucleated "sole" of the motor end-plate is largely an artefact, derived by expression of the interfibrillar substance of the muscle-fibre The mode of ending of the nerve-fibres in the muscle-spindles shows them, in agreement with Sherrington's experiments, to be sensorial end-organs. The methylene-blue method bears out the description of the ending given by Ruffini, and reveals further details of considerable interest.

Prof. K. Huerthle described a method by which the resistance offered by the vascular channels of, an organ to the blosd. flow through them may be measured. The viscosity of the blood which, together with the dimensions of the tubular system, form the two factors on which the resistance depends, was determined by allowing the blood from an artery to flow through calibrated capillary tubes for thirty seconds, the quantity, pressure, and duration of flow being accurately measured. The internal friction of the blood of the dog is about 4.5 and of the rabbit $3 \cdot 2$, that of water being unity. If in any particular organ 
the quantity of blood flowing through in a unit of time, the arterial pressure, and the coefficient of viscosity be known, it is easy by Poiseuille's law to calculate the bore and length of a tube through which, under given conditions, the same quantity of blood would flow. The aim of the experiments is to measure and express the resistance through the several regions and organs of the body in this manner.

Prof. A. R. Cushny described rhythmic variations in the strength of the contractions of the mammalian ventricle under the action of certain drugs of the digitalis groups. The variations occur only when the ventricle is beating at a rate different from that of the auricle. If the conjunction of the auricular with the ventricular beat fall at a favourable time, the beat of the ventricle is strong; if at an unfavourable, is weak. The systole of the auricles at a certain stage of the rhythm falls at the systole of the ventricle : the auricle in consequence cannot empty itself. It was also pointed out that the action of nicotin on the mammalian heart is exactly similar to its action as discovered by Langley on the ganglia of the sympathetic system.

Prof. Loeb demonstrated experiments on the influence of the discharge of highly-charged conductors on nerve-muscle preparations. It was known that if a highly-charged conductor be discharged in the neighbourhood of a nerve, the muscle contracts. He found in relation to this the following new facts. (I) If the nerve be placed parallel with the spark discharge of a friction machine, maximal effects are obtained when the electrodes are symmetrical in regard to the nerve. If the nerve be set to one side near one electrode the effects decrease. (2) If the nerve be set at right angles with the spark discharge, minimal effects are obtained when the electrodes are symmetrical to the nerve. Strong effects are obtained by placing the nerve near one electrode. (3) By putting a conductor behind the nerve in Case I, the effects are effaced or diminished. By putting a conductor between the nerve-muscle preparation and one electrode in Case 2, an increase in effect is produced. It had been shown in the last ten years that the phenomena of heliotropism, long observed in plants alone, are common in the animal kingdom. In both cases the effects of the light are determined by the direction of the rays and the length of waves, the more refractive of the visible spectrum being chiefly effective. It is possible to directly stimulate nerve by electric rays. Apparently the galvanic current does not directly act on living matter; its effects as stimulus being in reality only due to the direct or secondary products of electrolysis.

Prof. Waller gave a demonstration and description of the action of various reagents upon the electrotonic currents of nerve. He has succeeded in completely proving that these currents are in the strictest sense physiological, and they therefore become more than ever of high physiological interest. In the frog's nerve the anelectrotonic current considerably exceeds the katelectrotonic in magnitude. The action of acids, of alkalis, of carbonic acid, of some anzesthetics, and also of tetanisation and of variations of temperature were all dealt with in their bearing upon the ratio and magnitude of the electrotonic currents. Slight acidification diminishes the anelectrotonic current and increases the katelectrotonic: treatment with bases diminishes in a typical manner the katelectrotonic current. The effect of prolonged tetanisation upon the katelectrotonic current is similar to that of acidifidation. Its effect, though less uniform on the anelectrotonic current, yet strongly resembles the effect produced by carbonic acid. Prof. Waller, indeed, holds that his experiments bring strong evidence that the tetanisation of nerve induces in the nerve the production of $\mathrm{CO}_{2}$ to a thus detectible extent. Electrotonic currents are diminished for a time by exhibition of ether vapour, and are rapidly and permanently abolished by weaker percentages of chloroform vapour. In other words, chloroform rapidly kills the nerve outright, while by ether it is easy to merely diminish or abolish the excitability of the nerve for a temporary period.

Dr. Mackay communicated a paper on the absorption of "ferratin" and of hremoglobin by the intestinal wall. He had used the microchemical tests for iron devised by Prof. Macallum. He found that the iron of ferratin is absorbed by the epithelial cells of the villi, and that it was passed inwards to the leucocytes. Deposits of iron could further be found in the liver, spleen, and lymph glands. The iron of hremoglobin is absorbed by the epithelial cells as a compound, probably hæmatin. In this case deposits are also found in the organs of the portal system, and there is indication of some excretion of iron in the bile and in the urine.

Dr. Noël Paton read a paper on the phosphorus metabolism of the salmon in fresh water. Sample salmon were throughout the spring, summer and autumn taken from the mouths of certain rivers, and others from the upper waters of the same rivers. Abundant observations clearly show that the fish do not feed during their stay in fresh water. The muscle substance steadily diminishes, while the ovaries and testes grow at its expense. The fats and proteids lost from the muscles are sufficient to supply these materials for the growing genitalia, and to yield a very large amount of energy for muscular work. The question here discussed is the exchange of phosphorus. It is first shown that in muscle the phosphorus is chiefly in the form of inorganic phosphates, though a comparatively large amount of lecithin and a small amount of nuclein are also present. In the ovary the phosphorus is chiefly combined in the pseudo-nucleinichthulin; but it is also present in considerable amounts in lecithin, and in very small amounts as inorganic phosphates. In the testis the phosphorus is chiefly in the form of true nucleins, but there are also a considerable quantity of lecithin and a small quantity of inorganic phosphate. As the season advances the phosphorus in the genitalia increases, while the phosphorus of the muscle diminishes. The loss of phosphorus from the muscle is barely sufficient to account for the gain in the ovary, amply sufficient to yield the increase of phosphorus in the testis. The lecithin lost from the muscle is sufficient only to account for a small part of the lecithin gained by the ovary. The lecithin and ichthulin of the ovary must thus be found by synthesis as these structures grow. The nuclein of the testis must be formed in a similar manner.

The presence of considerable amounts of lecithin in the growing ovary and testis would seem to indicate that this substance is one of the first stages in the construction of nucleo compounds.

Prof. W. P. Lombard gave a communication on the effect of frequent excitations on the contractility of striped muscle. When the muscle is made to give a series of contractions the height of the contraction is seen to increase. The muscle contracted very much higher to an induction shock just after than just before a short period of tetanic excitations. Each of a series of short tetani following each other in rapid succession was, until fatigue appeared, higher than its predecessor. The after effect of excitation is to increase the capacity of the muscle to shorten. When the inertia of the writing lever is increased each of a series of short tetani is seen to begin with a sudden high rise, the throw of the lever carrying the curve above the actual shortening of the muscle. The height of the introductory peak exhibits in repeated tetani a staircase-like growth, and this, in spite of the total contraction being lessened by an increasing growth of contracture heightening the base-line. The effect of the repeated excitations is to cause a more and more sudden and intense liberation of energy.

Prof. Sherrington demonstrated the production of an intense colour of subjective origin by whirling a disc coloured with red on a black-white ground. The disc should contain a black sector of about $170^{\circ}$. From one edge of the black sector broad concentric vermilion arcs are carried into the peripheral white part of the discs for about $90^{\circ}$; from the other edge of the disc similar arcs in the central part of the white field. On rotating the disc so that black follows red in the circumferential field, and precedes red in the central, the outer arcs appear a dull dark maroon, the inner a bright orange, and the spaces between the outer arcs appear deep blue-green, and the spaces between the inner arcs appear primrose-yellow, especially when viewed by a yellowish illumination. Prof. Sherrington offered an explanation for the phenomenon which he based on negative afterimages heightened by simultaneous and successive contrasteffects which, as he demonstrated at the meeting. take effect even when speed of translation of a surface prevents the mind from perceiving the space-relations of the distribution of the contrasted tints. It was pointed out that the disc is being used in this experiment similarly to a circular rheotome for summation of effects which taken singly would by reason of their small quantity be imperceptible. It was also demonstrated that by whirling the disc at higher speed the phenomenon becomes altered, the reds and greys then matching over the whole surface of the disc, the greys assuming a pale green tint; when this is obtained, it was urged that one factor

$$
\text { Ni) 1462, vOL. 57] }
$$


of those previously acting, namely simultaneous contrast, had alone become valent.

Prof. Halliburton and Dr. Mott made a communication on the effects produced on the arterial blood-pressure by the intravenous injection of choline, neurine, and allied substances. Normal cerebro-spinal fluid produced no effect, while that obtained postmortem from cases of general paralysis of the insane produced a fall of blood-pressure. This is not due to a proteid body present ; for after coagulation by boiling, or precipitation by alcohol, a similar effect is produced. Neurine hydrochloride in $O \cdot I$ per cent. solution gave a similar fall, but in most cases this was followed by a rise and then by a more persistent fall of blood pressure. Choline hydrochloride in 0.2 per cent. solution gave results identical with those obtained by the pathological cerebrospinal fluids. Blood taken from patients suffering from pseudoapoplectiform convulsions of general paralysis was precipitated with alcohol, and the filtrate, after evaporation to dryness and solution in saline, injected, and the effect produced corresponded entirely with that obtained with pathological cerebro-spinal fluids and with solution of choline. Normal blood gave a negative result. The fall of blood-pressure produced by these various reagents is cardiac in origin. This was established by plethysmographic tracings and by experiments on the frog's and mammal's heart. This agrees very well with what is found in general paralysis, cardiac weakness, and enfeebled circulation.

Prof. Richet related experiments by which he had succeeded in showing and measuring in the dog the refractory period of the bulbar and cerebral nervous centres. The animal is cooled down to $30^{\circ} \mathrm{C}$. and anæsthetised. It will then react to electrical stimulations of the cortex if the stimulations be not too frequent. If the rhythm of these be I per second, the responses are equal in magnitude ; if 4 per second, there will be one large and then one small response ; if ro per second, there will be no longer a response to each excitation but one to each alternate. Half of the stimuli fall within a refractory period. The duration of the refractory period is $1 / \mathrm{IO}^{\prime \prime}$. It is possible to show that, as was originally indicated by the experiments of V. Kries, volitional impulses have a frequency of repetition of about 10 or $I I$ in a second.

Dr. F. S. Lee brought forward the results of his continued researches into the functions of the semicircular canals in fishes, especially in regard to maintenance of equilibrium and to locomotion.

Prof. Anderson Stuart made an interesting communication on the canal of Stilling. He also showed models illustrating the horopter.

Mr. O. Grunbaum contributed a communication upon the effects of intermittent retinal stimulation. He exhibited the results of several series of experiments stated in curves with measures of speed of alternation as ordinate-heights and degrees of illumination as abscissæ. The curves so obtained possess each an apex above, denoting that a sensation of continuous stimulation results if the luminosity be below or beyond a certain amount. The curve begins to descend some distance prior to the use of a degree of luminosity such as to produce a blinding after-image.

Dr. A. Grunbaum gave a communication on muscle spindles found in human muscles; the communication was illustrated by microphotograms.

Profs. Boyce and Herdman contributed the results of their investigations on "green oysters." They had demonstrated copper in comparatively large quantity in the green leucocytes of the American oyster. The green colour in these oysters is in direct proportion to the copper present. The copper indicates a pathological condition of these American oysters. They were not prepared to state whether copper in the food of the oyster can bring about the condition, but there is abundant evidence to show that it can occur where no copper mines or other evident sources of copper are present. The normal copper of the hremocyanin of the blood, which is probably constantly circulating through the body in minute quantity, may cease to be removed, and so become stored in certain cells in the oyster. The deposition of the copper in the large quantity found appeared to then the result of a degenerative process.

Prof. Boyce communicated a paper by Dr. Warrington (Liverpool). The effects of ischomia on the structural features of nerve-cells were shown to be very marked. Also the effect of cutting off from the anterior coronal cells the afferent impulses usually impinging on them was studied in cases where the posterior roots had been divided. Marked changes were found, which were minutely described. On the other hand, attempts to discover changes in the cells of the oculomitorius and facialis nuclei after section of their nerve-trunks failed to detect any changes.

In experiments of the kind mentioned above, in which changes uniformly resulted, the typical picture of alteration is very charac. teristic ; the cell becomes somewhat enlarged, is stained red, with a small amount of blue chromophitic granules at its periphery, the nucleus remaining well-marked. The changes go on to a further swelling up of the cell, a disappearance of its nucleus, and finally shrinkage and ultimate disappearance. The paper was illustrated by microphotographs. The method of staining of the preparations employed had been the methylene-blue and erythrosin modification of Nissl's stain.

Prof. Macallum made a long and important communication on the structures of the nucleus and body of the cell, and described the views at which he had arrived on this profound and difficult subject.

Prof. Wesley Mills contributed interesting papers on the psychic development of young animals, on the functional development of the cerebral cortex, and on cortical cerebral localisation.

Miss F. Welby contributed an interesting account of observ. ations on the effect of curvesthetic vapours on the cardiac muscle of the frog; her remarks being illustrated by the projection of the graphic records of the experiments.

The morning of Tuesday, August 24, was devoted to a com. bined meeting of the sections Physiology and Botany to discuss the chemistry and structure of the cell. Several members of the section of Chemistry also took part. the opening paper being by Prof. Meldola. Profs. Marshall Ward, Armstrong, Green, Macallum, Remsen, Farmer, and Halliburton, spoke in the discussion.

Prof. Meldola devoted his opening paper to a discussion of the rationale of chemical synthesis of bodies formed by living organisms.

The presidential address by Prof. Foster was given in the large Zoology Theatre of the University; it was very numerously attended. It was a retrospect of the history of physiology since the previous meeting of the British Association at Montreal in I 884 . It was pointed out that the opportunities for studying physiology had grown larger and more facile. "But there is still a larger outcome from the professional chair and physiological laboratory than the training of students. Each post for teaching is no less a post for learning. Among academic duties the making of knowledge is no less a duty than the distributing of it." "Practical expression has been given to this feeling more vigorously in Canada and the United States than in the old country." "Physiology is destined, in consequence of its containing the study of the actions of the brain, to modify the attitude of the physiologist toward the world, and of the world toward the physiologlst. That physiology is, and must always be the basis of the art of healing is a truism, but if a plebiscite limited to instructed, one might almost say scientific, men were taken at the present moment, it would probably enough be found that the most prevalent conception of physiology is that it is something which is in some way an appendage to the art of medicine." But without plunging " "into the deep waters of the relation which body bears to mind, this at least stares us in the face that changes in what we call the body bring about changes in what we call the mind. When we alter the one we alter the other. . . If, as the whole past history of our science leads us to expect, in the coming years a clearer and clearer insight into the nature and conditions of that molecular dance which is to us the material token of nervous action, and a fuller exacter knowledge of the laws which govern the sweep of nervous impulses along fibre and cell, give us wider and directer command over the moulding of the growing nervous mechanism and the maintenance and regulation of the grown one, then assuredly physiology will take its place as a judge of appeal in questions not only of the body, but of the mind; it will raise its voice, not in the hospital and consulting room only, but also in the senate and the school."

\section{ON THE SUMMIT OF MAUNA LOA.}

$D^{\text {R. H. B. GUPPY has contributed to the Pacific Commercial }}$ Advertiser, published at Honolulu, an account of observ. ations made during a three weeks' sojourn upon the summit of Mauna Loa in August last.

The air at first was highly electrified. A red blanket used No. I 462 , VOL. 57 ] 